\title{
Prevalência de cárie associada à escolaridade materna e ao nível socioeconômico em escolares
}

\author{
Prevalence of childhood dental caries and its association between mothers' level of schooling and socioeconomic \\ status of family in schoolchildren
}

Aline Scapinello

Curso de Graduação em Odontologia, Faculdade da Serra Gaúcha, Caxias do Sul, Rio Grande do Sul, Brasil

Estelamari Barbieri Elsemann

Professora Mestre do Curso de Odontologia, Faculdade da Serra Gaúcha, Caxias do Sul, Rio Grande do Sul, Brasil

Rogério Brasiliense Elsemann

Professor Doutor de Prótese do Curso de Odontologia, Faculdade da Serra Gaúcha, Caxias do Sul, Rio Grande do Sul, Brasil

Henrique Sangoi

Cirurgião-Dentista, Prefeitura Municipal da Saúde, Município de Nova Roma do Sul, Rio Grande do Sul, Brasil

\section{Alexandra Flávia Gazzoni}

Professora Pós-doutora do Curso de Odontologia

Laboratório de Microbiologia e Patologia Bucal, Faculdade da Serra Gaúcha, Caxias do Sul, Rio Grande do Sul, Brasil

- Os autores declaram que não há conflito de interesse.

\section{Resumo}

Objetivo: Este estudo avaliou a prevalência de cárie e sua associação com escolaridade materna e índice socioeconômico em escolares de Nova Roma do Sul (RS). Material e Métodos: Foram avaliadas 120 crianças de 7 a 10 anos pertencentes a duas escolas do município para compilar índice CPO-D e ceo-d. Foi enviado às mães um questionário para avaliar escolaridade materna e renda familiar. Resultados: A prevalência da cárie foi $2,45 \%$. A média de CPO-D foi $0,41 \pm 0,83$, enquanto a média de ceo-d foi $2,94 \pm 2,96$. Observou-se que tanto CPO-D quanto ceo-d não apresentaram associação com escolaridade materna e renda familiar, porém valores de ceo-d foram altos. Conclusão: Resultados apontam que a alta prevalência de cárie no estudo independe da escolaridade materna e do nível socioeconômico das famílias.

Palavras-chave: epidemiologia; saúde pública; índice CPO.

\section{Abstract}

Objective: The aim of this study was to evaluate the prevalence of dental caries and investigate its association with socioeconomic status of family and parents's education among schoolchildren in Nova Roma do Sul, Rio Grande do Sul. Material and Methods: We evaluated 120 schoolchildren aged 7 to 10 years old belonging to two schools of this city to be evaluated for the DMFT index (dental caries) and early chilhood caries (ECC) by clinical examination. Socioeconomic data (years of education of the mothers and family income) were collected through a questionnaire that was completed by the mothers. Results: The prevalence rate of dental caries was $2,45 \%$. The mean DMFT index was $0.41 \pm 0.83$, while the mean ECC was higher and equal to $2.94 \pm 2.96$. Both the DMFT index and ECC there were no statistically significant associated with socioeconomic indicators, since the rates found for DMFT were high. Conclusion: Results indicate that high prevalence rate was an independent factor of the maternal education and socioeconomic status of families.

Keywords: epidemiology; public health; DMFT index.

\section{Introdução}

A cárie é uma doença infectocontagiosa, de caráter crônico, causada pelo processo de desmineralização da superfície dental por ácidos orgânicos. ${ }^{1-3}$ Estudos epidemiológicos nacionais realizados entre 1986 e 1996 constataram uma redução dos índices de cárie dentária. Surpreendentemente, verificou-se que esse declínio ocorreu acompanhado de um fenômeno conhecido como polarização da doença, a qual se caracteriza pela concentração dos mais altos índices da cárie em pequenos grupos populacionais. ${ }^{1,2,4}$

A renda familiar e o grau de escolaridade dos responsáveis pelas famílias estão diretamente relacionados às altas taxas de prevalência, bem como ao grau de severidade de cárie dentária na população infantil ${ }^{4}$. Dados retrospectivos revelam uma forte associação entre cárie dentária e estrutura socioeconômica e educacional das famílias. ${ }^{13-5}$ Destaca-se, assim, a importância de identificar as características sociodemográficas da população e associá-las ao desenvolvimento da cárie na população infantil. ${ }^{2,4}$

No que diz respeito à distribuição sociodemográfica do estado do Rio Grande do Sul (RS), Nova Roma do Sul é um município localizado na região nordeste do referido estado. Conforme dados da última atualização censitária, o município tem uma população de aproximadamente 3.343 habitantes, sendo que destes, $52,5 \%$ residem na zona rural e $47,4 \%$ na zona urbana. ${ }^{6}$

De acordo com levantamento epidemiológico em âmbito estadual, até o momento não há estudos realizados na região serrana do RS que objetiva avaliar relação entre cárie dentária, renda familiar e grau de escolaridade das famílias. Sendo assim, o presente estudo teve como objetivos avaliar a prevalência de cárie, associá-la a escolaridade materna e a renda familiar, em crianças de 7 a 10 anos pertencentes às duas escolas do município, as quais são consideradas por abrigar 100\% dos estudantes da cidade.

\section{Material e Métodos}

O estudo utilizou um delineamento de caráter transversal a partir de uma análise de dados de informações obtidas em um questionário denominado de Critério Brasil.?

Participaram deste estudo as duas únicas escolas públicas do município, sendo que uma delas localizava-se na área rural (Escola 1) e a segunda escola (Escola 2) pertencia a área urbana da cidade. 
Após a assinatura do Termo de Consentimento Livre e Esclarecido (TCLE), o questionário foi aplicado às mães dos escolares com idade entre 7 e 10 anos. O referido questionário foi validado pela Associação Brasileira de Empresas de Pesquisa (ABEP).

Com relação aos níveis socioeconômicos e escolaridade materna, as famílias foram classificadas conforme descrito nas Tabelas 1 e 2, respectivamente. Além da aplicação do referido questionário às mães, este trabalho também foi baseado em um instrumento de intervenção oral. Para tanto, os escolares foram submetidos ao exame oral, sendo utilizados os critérios propostos pela Organização Mundial da Saúde (OMS) para determinação da condição dentária. Deste modo, utilizou-se para avaliação o índice de dentes permanentes cariados, perdidos, obturados ou restaurados (CPO-D), e pela quantificação de dentes decíduos cariados, extrações recomendadas, obturados ou restaurados (ceo-d). ${ }^{8}$

Os exames bucais foram realizados por um único examinador treinado e calibrado previamente ao estudo e um anotador. Uma vez que os exames bucais exigem a manutenção de uma boa reprodutibilidade das observações, divergências de diagnóstico podem ocorrer entre diferentes exames de um mesmo examinador ao longo do tempo (erro intraexaminador). Para tanto, em momento prévio, bem como ao longo da coleta de dados, o examinador realizou a concordância intraexaminador, sendo a reexaminação com $5 \%$ de sua amostra. O grau de concordância intraexaminador da coleta de dados foi calculado através do coeficiente de Kappa (k).

Antes da realização do exame oral, foi preconizado o preenchimento do Termo de Assentimento Livre e Esclarecido (TALE). Para tanto, foi pedido para que cada criança assinalasse alguma categoria da Escala Hedônica de Expressão Facial, a qual é dividida em cinco categorias. Por sua vez, a criança, a qual assinalou a categoria identificada como sorridente foi incluída no estudo. Sendo assim, para a inclusão no estudo, tanto a mãe quanto a criança deveriam consentir sua participação.

Os exames intraorais foram realizados em locais que permitissem o uso da luz natural. Os escolares foram posicionados conforme a metodologia relatada por Matewson. ${ }^{9}$ Com auxílio de luvas descartáveis e espelho oral plano, foram utilizados abaixadores de língua e compressas de gaze estéril para auxiliar no afastamento dos tecidos, secar as superfícies dos elementos dentários e remover o biofilme dentário, respectivamente.

Os dados do presente trabalho foram analisados pelo Graph Pad Prism 5.0 (Graph Pad Software Inc., La Jolla, Ca). Para análise da associação entre índice de cárie/escolaridade materna e renda familiar utilizou-se o teste de Qui-quadrado $(\chi 2)$. Os resultados do CPO-d e ceo-d foram analisados utilizando teste $T$ de Student. Neste estudo foi adotado um intervalo de confiança (IC) de $95 \%$. Os dados foram considerados estatisticamente significantes quando o $P$-valor foi $\leq 0,05$.

O presente trabalho foi realizado de acordo com a Declaração de Helsinki (2008), bem como em concordância com a legislação vigente (466/12) do Comitê Nacional de Ética em Pesquisa e Conselho Nacional de Saúde (CONEP/CNS) e suas complementares. O referido trabalho foi aprovado pelo Comitê de Ética em Pesquisa da Faculdade da Serra Gaúcha (CEP/ FSG), sob CAAE de no 47947015.1.0000.56.

Tabela 1. Estimativas para renda domiciliar mensal para os estratos socioeconômicos*

\begin{tabular}{|c|c|c|}
\hline Classes & Pontos & Renda Média Domiciliar \\
\hline A & $45-100$ & $20.272,56$ \\
\hline B1 & $38-44$ & $8.695,88$ \\
\hline B2 & $29-37$ & $4.427,36$ \\
\hline C1 & $23-28$ & $2.409,01$ \\
\hline C2 & $17-22$ & $1.446,24$ \\
\hline D e E & $0-16$ & 639,78 \\
\hline
\end{tabular}

*Fonte: Critério Brasil (2015)ㄱ.
Tabela 2. Grau de instrução da mãe, segundo Critério Brasil (2015)*

\begin{tabular}{l|c|}
\multicolumn{1}{|c|}{ Escolaridade da mãe } & Pontuação \\
\hline Analfabeto / Fundamental I incompleto & 0 \\
\hline Fundamental I completo / Fundamental II incompleto & 1 \\
\hline Fundamental II completo / Médio incompleto & 2 \\
\hline Médio completo / Superior incompleto & 4 \\
\hline Superior completo & 7 \\
\hline *Fonte: Critério Brasil $(2015)^{7}$.
\end{tabular}

\section{Resultados}

O cálculo do coeficiente de Kappa para a concordância intraexaminador em período prévio ao estudo foi 0,93 ( $k a p p a=$ $0,93)$. Ao longo da coleta de dados, alcançou-se a média de $0,90(k a p p a=0,90)$, indicando que a concordância intraexaminador foi alta em ambos os momentos.

No total, 120 escolares foram examinados por meio do exame oral, uma vez que os mesmos tiveram os respectivos questionários devolvidos, bem como as devidas autorizações pertinentes ao estudo, e assim representaram a população final de estudo.

Do total, 50 escolares $(41,6 \%)$ eram do gênero feminino e 70 (58,3\%) eram do gênero masculino. A média de idade, para ambos os sexos, foi de 8,62 anos $(7-10 ; \pm 1,11)$. No sexo feminino, a média de idade foi de 8,97 anos $(7-10 ; \pm 1,0)$. No sexo masculino, a média foi menor e igual a 8,67 anos $(7-10 ; \pm 1,12)$. Quando comparada idade entre os grupos feminino e masculino, não foi observada diferença significativa entre os grupos estudados $(P=0,08)$. 


\section{- Características Sociais}

Em relação aos dados coletados por meio do questionário, foi constatada a participação de crianças pertencentes aos seis níveis socioeconômicos propostos (Figura 1), sendo a maior parte das crianças pertencentes as Classes B2 com 34 (28\%) escolares e $\mathrm{C} 1 \mathrm{com} 40$ (33\%). De acordo com a Figura 2, quando avaliada a escolaridade materna total do estudo, os valores encontrados foram os seguintes: 29 (24\%) mães eram analfabetas ou tinham fundamental incompleto, 41 (34\%) delas pertenciam ao grupo fundamental I completo/Fundamental II incompleto, 18 (15\%) mães faziam parte do grupo fundamental II completo/médio incompleto, 18 (15\%) tinham ensino médio completo ou superior incompleto e 14 (12\%) estudaram o ensino superior completo.

\section{- Características Encontradas Relacionadas às Instituições de Ensino Escola 1}

Nesta instituição de ensino foram avaliados 70 escolares. Do total examinado, $29(41,4 \%)$ eram meninas e 41 (58,5\%) eram meninos. A média de idade obtida em relação às meninas e aos meninos foi de $8,72( \pm 1,16)$ e $8,78( \pm 1,06)$, respectivamente. Foram avaliadas 70 famílias, onde o maior número de famílias (Figura 1) nesta escola foram consideradas como pertencentes da classe C1 (38,5\%), seguido da classe B2 (25,7\%). De acordo com a Figura 2, o maior número de mães foram classificadas no grupo Fundamental I completo/Fundamental II incompleto, com percentual encontrado de 37,1\%.

Com relação aos dados originados do Îndice CPO-D, o mesmo apresentou uma média de $0,78(0-3 ; \pm 0,76)$. Quanto aos resultados observados no ceo-d, a média obtida foi considerada alta, sendo igual a $2,94(0-11 ; \pm 2,96)$. Em relação à prevalência de cárie dentária, o valor encontrado foi de 3,32\%. Na dentição decídua, a prevalência foi de $2,9 \%$, enquanto na dentição permanente a prevalência encontrada foi de $0,38 \%$. A observação das distribuições do ceo-d e seus componentes segundo os quartis permitem visualizar que $75 \%$ das crianças desta escola apresentam ceo-d, entre 4,25 e 11, o qual é caracterizado como um índice alto. Sendo assim, observa-se que a distribuição deste índice não é normal.

Com respeito à categorização do Índice de $\mathrm{CPO}-\mathrm{D}$ em um grupo que não possui cárie $(\mathrm{CPOD}=0)$ e um grupo que possui cárie $(\mathrm{CPOD} \geq 1)$, permite-se observar que quando avaliada a associação entre Índice CPO-D e escolaridade materna, não foi observada diferença significativa entre os grupos estudados $(P=0,06)$, resultado estatístico igualmente encontrado quando foi avaliada a associação entre ceo-d e escolaridade materna $(P=0,45)$.

Em relação à existência de associação entre as variáveis Índice $\mathrm{CPO}-\mathrm{D} /$ nível socioeconômico e ceo-d/nível socioeconômico também não foi encontrada diferença significativa entre os escolares pertencentes a Escola $1(P=0,37$ e $P=0,18$, respectivamente).

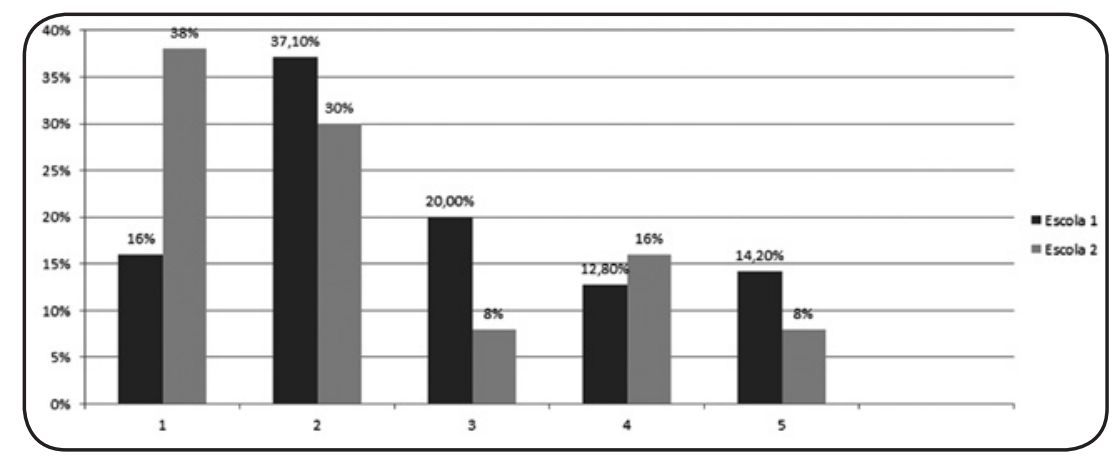

Figura 1. Distribuição das porcentagens da escolaridade materna encontradas na Escola 1 e Escola $2(n=120)$

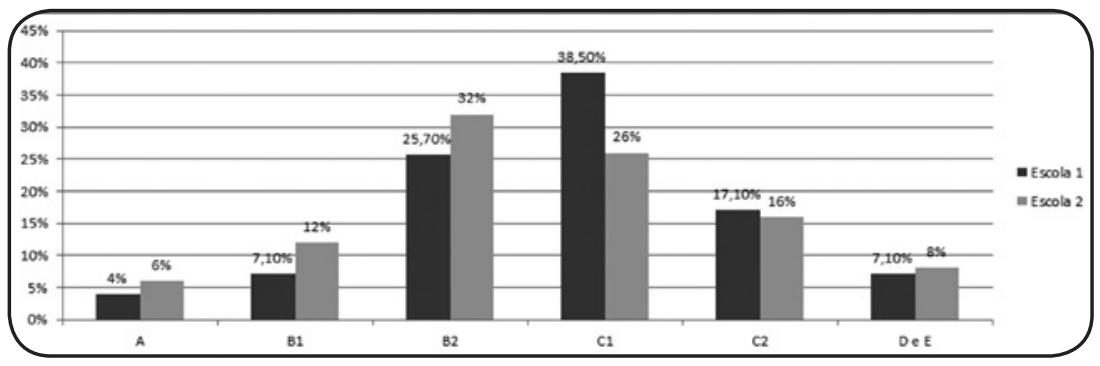

Figura 2. Distribuição dos níveis socioeconômicos encontrados no estudo em ambas as escolas $(n=120)$ 


\section{Escola 2}

Do total de 50 crianças examinadas nesta escola, 21 (42\%) eram meninas e 29 (58\%) eram meninos. A média de idade obtida para as meninas e meninos foi de 8,57 $\pm 1,13 \mathrm{e}$ $8,27 \pm 1,22$, respectivamente. Foram avaliadas 50 famílias, observando que um maior número de famílias pertencia à classe C1 (26\%), seguido da Classe B2 (32\%) (Figura 1). Conforme os resultados encontrados (Figura 2), identificou-se que a maior porcentagem das mães com filhos estudando na Escola 2 foram classificadas dentro do grupo analfabeto/fundamental I incompleto, apresentando um percentual igual a $38 \%$.

O Índice de CPO-D apresentou uma média de 0,46 \pm 0,93 . Com relação aos resultados encontrados no ceo-d, a média obtida foi de $1,16 \pm 2,11$. Nesta escola, foi observada uma prevalência total de cárie dentária igual 2,12\%. Na dentição decídua, a prevalência foi de 1,66\%, já na dentição permanente a prevalência encontrada foi de $0,46 \%$. A observação das distribuições do ceo-d e seus componentes segundo os quartis permite observar que a distribuição deste índice também não é normal, com 75\% das crianças apresentando ceo-d entre 3 e 11. Estes dados são considerados altos e igualmente foram encontrados na Escola 1.

Do cruzamento de dados após a coleta, quando foi avaliada a associação entre Índice CPO-D e escolaridade materna não foi observada diferença estatisticamente significativa $(P=1,0)$. Do mesmo modo, ao analisarmos a existência de associação entre ceo-d e escolaridade materna, também não foi demonstrada diferença estatisticamente significante $(P=0,58)$. Em relação à avaliação da associação entre Índice CPO-D e nível socioeconômico, bem como ceo-d e nível socioeconômico, constatou-se que não foi verificada nenhuma diferença significativa entre as variáveis avaliadas $(P=0,62$ e $P=0,58$, respectivamente).

\section{- Prevalências Totais do Estudo}

Enquanto a média total do estudo para o índice CPO-D foi de $0,41 \pm 0,83$, a média total de ceo-d foi maior e igual a $2,94 \pm 2,96$. A prevalência total de cárie dentária do estudo levando consideração os dados registrados nas duas escolas foi de 2,45\%. Em relação às duas instituições e o Índice CPO-D, não foram encontradas diferenças significativas $(P=0,63)$. Quando avaliado o índice ceo-d, foi encontrado diferença significativa ( $\mathrm{P} \leq 0,001)$, uma vez que as médias encontradas para ceo-d foram para a escola 1 de 2,9 e para a escola 2 de 1,6.

Quando foi testada a associação entre escolaridade materna de ambas as escolas e Índice CPO-D, não se verificou diferença estatisticamente significativa $(P=0,10)$. Também não foi constatada diferença significativa quando foi avaliada a associação entre ceo-d e escolaridade materna entre ambas escolas $(P=0,39)$.

Em relação à avaliação estatística de associação entre índice CPO-D e nível socioeconômico, não se observou diferença significativa $(P=0,70)$. Da mesma forma, quando foram comparadas valores ceo-d e nível socioeconômico de ambas escola, também não foi constatada diferença significativa $(P=0,54)$.

\section{Discussão}

A cárie ainda é um problema de saúde pública, apontada como principal causa de perda dentária. Apesar dos esforços realizados, ela ainda é considerada uma endemia mundial, principalmente entre crianças e adolescentes. ${ }^{10}$ Esta condição clínica é considerada uma doença polarizada, na qual apresenta-se com maior necessidade de tratamento e com maior prevalência na população menos favorecida. ${ }^{4,11}$ Entretanto, observando os resultados encontrados não é possível afirmar, a partir do nosso estudo, esta polarização da doença, uma vez que a proporção de crianças com altos índices CPO-D e ceo-d é igualmente alta tanto naqueles escolares, nos quais as mães têm escolaridade materna maior a 8 anos, bem como naqueles escolares, que pertencem a níveis socioeconômicos mais altos.

Em relação aos níveis encontrados do CPO-D, os valores encontrados são baixos em nosso estudo. Este aspecto reafirma os resultados de Narvai et al., ${ }^{12}$ o qual também encontrou redução do Índice CPO-D. De fato, este dado está atribuído à expansão da fluoretação das águas de abastecimento público, principalmente nas regiões Sul e Sudeste no país. Vale ressaltar que no município de Nova Roma do Sul, a população pesquisada também tem acesso a água fluoretada, uma vez que a efetividade da fluoretação das águas no declínio da cárie é um fato amplamente aceito, tanto por especialistas em saúde pública quanto pela comunidade odontológica, no Brasil e em âmbito internacional.

Em relação ao ceo-d, os valores encontrados em nosso estudo são maiores àqueles encontrados no último SBBRASIL, ${ }^{13}$ realizado em 2010, o qual afirma que a média encontrada para a região Sul foi de $2,49 \%$. Nosso estudo apresenta índices maiores de ceo-d quando comparados aos valores encontrados pelo SBBRASIL nas regiões Sudeste e Nordeste do país. Nossos resultados são similares a região Centro-Oeste. Em contrapartida, são inferiores quando comparados aos resultados da região Norte do Brasil.

Em relação à avaliação de CPO-D, ceo-d e escolaridade materna em ambas escolas, não foi encontrada significância estatística neste estudo. Nossos dados discordam de um estudo realizado no estado de Amazonas por Viana et al. ${ }^{14}$, o qual refere que indivíduos com ensino fundamental incompleto e renda familiar inferior a cinco salários mínimos apresentaram as piores condições em relação à cárie dentária. Fato que nos leva a refletir não somente sobre a importância do nível de educação dos pais, mas também a influência do acesso de políticas públicas de saúde oral da população. Quando avaliados CPO-D e ceo-d associados em nível socioeconômico em ambas escolas, observou que não houve influência do nível socioeconômico nos valores encontrados. Nossos resultados suportam àqueles publicados por Gonçalves et $a l .{ }^{10}$, no qual constatou-se a ausência de associação entre a renda familiar e a prevalência de cárie. Em contrapartida, outros estudos realizados também no Brasil associam a 
renda das famílias com prevalência de cárie. ${ }^{1,2,4}$ Entretanto, essa diferença de dados deve-se a distintas metodologias aplicadas na análise dos resultados.

Nossos resultados são de significativa importância, visto que crianças que apresentam altos índices de cárie dentária têm maior probabilidade de desenvolver cáries subsequentes na dentição na permanente. Ainda destaca-se que as consequências dessa enfermidade influenciam na saúde como um todo. Tais alterações são observadas quanto ao crescimento mais lento das crianças quando comparadas às crianças livres de cárie, bem como na apresentação de baixo peso devido à associação da dor ao ato de comer. ${ }^{3}$

Neste contexto, aqui nós constatamos a alta prevalência de cárie dentária encontrada independe da escolaridade materna, bem como dos níveis socioeconômicos da população analisada. Fato que nos remete à falta de política pública relacionadas à saúde oral na população-alvo. Em adição a isso, a partir dos resultados observados em nosso estudo, contata-se a falta de planejamento em relação à saúde oral da população. Assim, novos estudos deveriam ser realizados para conhecer outros fatores predisponentes para o aparecimento da doença, bem como criar soluções para garantir uma melhor qualidade de vida da população.

\section{Conclusão}

De acordo com os resultados encontrados em nosso estudo, pode-se concluir que a renda familiar e a escolaridade materna não influenciaram na prevalência de cárie das crianças avaliadas, uma vez que o valor encontrado é considerado alto tanto em crianças pertencentes a classes $\mathrm{A}$ e $\mathrm{B}$, bem como naquelas crianças cujas mães apresentam oito ou mais anos de escolaridade. Dessa forma, nosso estudo reafirma a ausência de cobertura adequada de saúde oral nessa localidade, até mesmo para os indivíduos que, em teoria, poderiam custear seu tratamento. 


\section{Referências ::}

1. Peres KA, Bastos JRM, Latorre MRDO. Severidade de cárie em crianças e relação com aspectos sociais e comportamentais. Rev Saúde Pública. $2000 ; 34: 402-8$.

2. Ribeiro AG, Oliveira AF, Rosenblatt A. Cárie precoce na infância: prevalência e fatores de risco em pré-escolares, aos 48 meses, na cidade de João Pessoa, Paraíba, Brasil. Cad Saúde Pública. 2005;21:1695-700.

3. Parisotto T, Oliveira CS, Silva CMS, Almeida MEC, Rodrigues LKA, Santos MN. A Importância da prática de alimentação, higiene bucal e fatores socioeconômicos na prevalência da cárie precoce da infância em pré-escolares de Itatiba-SP. ROBRAC. 2010;19:333-39.

4. Silva ABVA, Oliveira LMC, Silveira RG, Miasato JM, Neves AA. Prevalência de cárie dentária em pré-escolares de uma escola particular em uma região rural do Estado do Rio de Janeiro. Rev Bras Epidemiol. 2012;14:49-56.

5. Freitas SFT, Lacerda JT, Neumann SRB. Prevalência de cárie e fatores associados na dentição decídua em escolares de sete anos de idade da rede pública de Joinville, Santa Catarina. Pesqui Bras Odontopediatria Clin Integr. 2010, 10:405-411.

6. Federação das Associações dos Municípios do RS. Porto Alegre: Portal Municipal. Índices Econômicos [citado 2015 Novembro 15]. Disponível em URL: HTTP://www.portalmunicipal.org.br/entidades/famurs/ dado_geral/

7. Associação Brasileira de Empresas de Pesquisa. São Paulo: Critério de Classificação Econômica Brasil [citado 2015 Junho 21] [6 páginas]. Disponível em URL: http://www. abep.org/

8. Nascimento S, Scabar LF. Levantamento epidemiológico de cárie utilizando os índices CPO-D, ceo-d e IHOS, nos índios da aldeia Wakri no Estado do Pará. J Health Sci Inst. 2008,26:247-54.

9. Mathewson RJ, Primosch RE, Robertson D. Fundamentals of dentistry for children. 1nd ed. Chicago: Quintessence; 1982. p. 81.

10. Gonçalves ER, Peres MA, Marcenes W. Cárie dentária e condições sócio-econômicas: um estudo transversal com jovens de 18 anos de Florianópolis, Santa Catarina, Brasil. Cad Saúde Pública. 2002;18:699-706.

11. Landim JR, Neta MCAF, Martins MCA, Nuto SAS, Braga JU. Fatores demográficos e socioeconômicos associados à cárie dentária em uma comunidade nordestina de baixa renda. RFO UPF. 2013;18:75-82.

12. NarvaI PC, Frazão P, RoncallI AG, Antunes JL. Cárie dentária no Brasil: declínio, polarização, iniquidade e exclusão social. Rev Panam Salud Publica. 2006;9:385-93.

13. Brasil. Ministério da Saúde. Secretaria de Atenção a Saúde. Departamento de Atenção Básica. Coordenação Geral de Saúde Bucal. Projeto SBBrasil 2010: 1 Pesquisa Nacional de Saúde Bucal - Resultados Principais. Brasília: Ministério da Saúde; 2010.

14. Viana ARP, Parente RCP, Borras MR, Rebelo MAB. Prevalência de cárie dentária e condições socioeconômicas em jovens alistandos de Manaus, Amazonas, Brasil. Rev Bras Epidemiol. 2009;12:680-7.

Recebido em: 04/04/2016 / Aprovado em: 25/04/2016

Alexandra Flávia Gazzoni

Departamento de Odontologia, Faculdade da Serra Gaúcha, Caxias do Sul

Rua Os dezoito do Forte, 2366, São Pelegrino

Caxias do Sul/RS, Brasil - CEP: 95020-472

E-mail: alexandra.gazzoni@fsg.br

Agradecimentos

Ao CNPQ. 